\title{
Oxymatrine suppresses MOVAS proliferation induced by platelet-derived growth factor via $G_{0} / G_{1}$ arrest.
}

\author{
Heng $\mathrm{Li}^{1}$, Ke Hu${ }^{1}$, Shan Gao ${ }^{1}$, Danna Tu${ }^{1}$, Jinbing You ${ }^{1}$, Xia Gao ${ }^{2 *}$ \\ ${ }^{1}$ Department of Pediatric Cardiovascular Medicine, Woman and Child Hospital of Hubei Province, Wuhan, Hubei, \\ PR China \\ ${ }^{2}$ Department of Pathology, Integrated Chinese and Western Medicine Hospital of Hubei Provincal, Wuhan, Hubei, \\ PR China
}

\begin{abstract}
Objective: The traditional Chinese medicine Oxymatrine (OMT) has numerous biological effects, such as anti-inflammatory, anti-fibrosis, anti-proliferation and anti-tumor. However, the underlying effect of OMT on vascular smooth muscle cells remains unclear. Herein, the aim of this study is to investigate the role and mechanism of OMT on MOVAS (a mouse vascular aortic smooth muscle cell line) proliferation induced by Platelet-Derived Growth Factor-BB (PDGF-BB).

Methods: Firstly, we assessed proliferation in MOVAS subjected to PDGF-BB induction or controlled intervention with/without OMT treatment. And then we detected cell cycle, cell apoptosis and the expression levels of cyclins, Cyclin-Dependent Kinases (CDKs) and p21 of each group.

Results: In the present study, we found that OMT remarkably restrained the proliferation induced by PDGF-BB stimulus. Additionally, it was demonstrated that cell population of MOVAS treated with OMT in the $S$ phase was reduced, but that of MOVAS in the G0/G1 phase was increased when compared to the PDGF-BB group. However, OMT treatment showed no effect on MOVAS apoptosis. Mechanistically, the expression of cyclinD1-CDK4/6 and cyclinE2-CDK2 were inhibited, while the expression of p21 was increased in MOVAS treated with OMT.

Conclusion: These results demonstrated that the inhibitory effect of OMT on proliferation of MOVAS were in part due to $G_{0} / G_{1}$ arrest by inhibiting cyclinD1-CDK4/6 and cyclinE2-CDK2, and promoting p21 expression. Therefore, OMT might become a new strategy for treating excessive proliferation of vascular smooth muscle cells in the future.
\end{abstract}

Keywords: Oxymatrine, MOVAS, PDGF-BB, Proliferation, Cell cycle.

Accepted on October 30, 2017

\section{Introduction}

Studies have shown that Vascular Smooth Muscle Cells (VSMCs) can secrete and release vascular regulating factors to maintain the physiological function of blood vessels [1,2]. However, excessive proliferation of VSMCs is the key pathophysiological process of neointima formation during atherosclerosis and post-angioplasty restenosis [3-5]. Thus, inhibition excessive proliferation of VSMCs is one of the important strategies to prevent restenosis after angioplasty. Platelet-Derived Growth Factor (PDGF) is an important regulator that stimulates the proliferation of VSMCs [6-8]. The binding of PDGF-BB, a subtype of PDGF, to its receptor triggers the activation of downstream signaling molecules such as Mitogen-Activated Protein Kinases (MAPKs) to promote VSMCs proliferation and growth [9-13]. Therefore, preventing the PDGF-BB induced VSMCs proliferation might be a promising strategy for treating the development of neointima in atherosclerosis and post-angioplasty restenosis in the future.
Oxymatrine (OMT), one of the main bioactive ingredients of Sophorae flavescentis radix, which is known as a traditional Chinese herbal medicine extracted from the dried root of Sophorae flavescens Aiton [14,15]. Previous studies have demonstrated that OMT shows a wide-range of pharmacological effects, including anti-inflammatory, anticancer, antiviral and immunomodulatory effects [14,16-19]. In recent years, the cardio-protective effects of OMT are becoming more and more arresting, and it has been used to treat cardiovascular diseases via anti-arrhythmic effect, vasodilative activity, hypolipidemic, positive inotropic effect and resistance of ventricular remodeling [20-25]. However, whether OMT exerts pharmacological effect on PDGF-BBinduced MOVAS (a mouse vascular aortic smooth muscle cell line) proliferation is not yet clear. Therefore, this study intends to explore the influence of OMT on MOVAS proliferation, and lay the theoretical basis for clinical intervention on atherosclerosis and post-angioplasty restenosis. 


\section{Materials and Methods}

\section{Materials}

Cell Counting Kit-8 was bought from Dojindo Molecular Technologies, Inc. (CK04-11, Rockville, Maryland, USA). TRIzol ${ }^{\circledR}$ Reagent was from Invitrogen Life Technologies

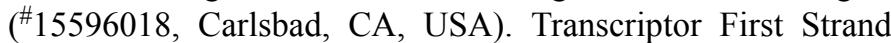
cDNA Synthesis Kit was purchased from Roche

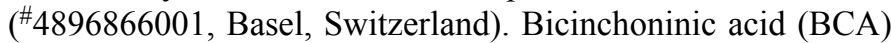
protein assay kit was from Thermo Scientific Pierce Biotechnology, Inc. ( ${ }^{2} 23227$, Rockford, IL, USA). Primary antibodies including anti-cyclinD1 antibody (\#2978, 1:1000),

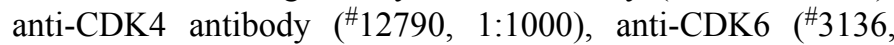
1:1000) antibody and anti-CDK2 antibody (\#2546, 1:1000) were from Cell Signaling Technology, Inc. (Danvers, MA, USA). Primary antibodies including anti-p21 antibody (sc-397, 1:500) and anti-cyclinE2 antibody (sc-22777, 1:500) were from Santa Cruz Biotechnology, Inc. (Dallas, TX, USA). Glyceraldehyde 3-phosphate dehydrogenase (GAPDH) (MB001, 1:10,000) was purchased from Bioworld Technology (St. Louis Park, MN, USA). HRP-conjugated goat anti-mouse $\left({ }^{\#} 074-1806, \quad 1: 10,000\right)$ or goat anti-rabbit $\left({ }^{\#} 074-1506\right.$, $1: 10,000)$ secondary antibodies were purchased from Kirkegaard and Perry Laboratories, Inc. (Gaithersburg, MD, USA). PDGF-BB was from Prospec-Tany Technogene Ltd. (CYT-501, Ness-Ziona, Israel). OMT was purchased from Shanghai Jinsui Biotechnology Co. Ltd. ("16837-52-8, Shanghai, China).

\section{Cell culture and treatment}

Briefly, the MOVAS cells (ATCC ${ }^{\circledR}$ CRL-2797TM), a mouse vascular aortic smooth muscle cell line, were cultured with DMEM/high glucose (SH30022.01, Hyclone) supplemented with $10 \%$ fetal bovine serum (SH30084.03, Hyclone), and 1\% penicillin-streptomycin (15140-122, ThermoFisher Scientific) at $37^{\circ} \mathrm{C}$. The cells were stimulated with $20 \mathrm{ng} / \mathrm{ml}$ PDGF-BB to induce MOVAS proliferation [26,27]. In the present study, to detect the appropriate concentration of OMT, the effects of several concentrations $(0,5,10,20,40 \mu \mathrm{mol} / \mathrm{L})$ of OMT on MOVAS proliferation were evaluated following exposure for $48 \mathrm{~h}$. Next, with the appropriate concentration, the cells were treated with different times $(0,12,24,48$ and $72 \mathrm{~h})$ to choose the appropriate treatment time. Upon the appropriate concentration and time of OMT, the cells were divided into the following groups: Phosphate Buffered Saline (PBS)+Dimethyl Sulfoxide (DMSO), PBS+OMT, PDGF-BB+DMSO and PDGF-BB+OMT.

\section{Cell proliferation assay}

Cell proliferation assay was performed according to the manufacturer's instructions of Cell Counting Kit-8 (CCK8). Firstly, cell suspension was inoculated in a 96-well plate and incubated. The MOVAS cells were then incubated with the CCK8 solution at $37^{\circ} \mathrm{C}$ for two hours. And then the absorbance of each well was measured at $450 \mathrm{~nm}$ (A450) by using a microplate reader.

\section{Cell flow cytometry analysis}

The MOVAS cells (about $2^{-5} \times 10^{6}$ cells) were collected by trypsin digestion, and these cells were washed with PBS for twice, then centrifugation to harvest cells. After re-suspending the cells with PBS, $5 \mathrm{ml}$ of pre-cold $70 \%$ ethanol was added to fix cells overnight at $4^{\circ} \mathrm{C}$. Next day, the cells were washed with PBS after discarding ethanol. Then, $0.3 \mathrm{mg}$ of Ribonuclease A (R5125, Sigma-Aldrich) and $0.015 \mathrm{mg}$ of PI (P4864, SigmaAldrich) were applied to stain cells in darkness. To avoid clumps, the stained cells were sorted by using BD FACS Aria ${ }^{\mathrm{TM}}$ III sorter for cell cycle analysis $(n=3)$.

\section{Real-time polymerase chain reaction (PCR) assay}

Briefly, total mRNA was extracted by using TRIZol reagent according to the manufacturer's instructions. Then, the cDNA synthesis was performed by using the Transcriptor First Strand cDNA Synthesis Kit. The relative mRNA levels of each genes were detected by CFX connect ${ }^{\mathrm{TM}}$ real-time $\mathrm{PCR}$ detection system (Bio-Rad) using $\mathrm{iQ}^{\mathrm{TM}} \mathrm{SYBR}^{\circledR}$ green supermix $(\mathrm{n}=4)$. $G A P D H$ gene expression was used as an internal control. The sequences of the primers pairs used in this study were designed by Primer 3.0 and displayed as follows: $G A P D H$ forward primer 5'-GGTGAAGGTCGGTGTGAACGGATTT-3' and GAPDH reverse primer 5' GCAGAAGGGGCGGAGATGATGA-3'; Ki67 forward primer 5'- CAGTACTCGGAATGCAGCAA-3' and Ki67 reverse primer 5'- CAGTCTTCAGGGGCTCTGTC-3'; PCNA forward primer 5'-CGTGTGGTGTGCTTTGGTAA -3' and PCNA reverse primer 5'-CCCAATACCACCACTCCACT-3'.

\section{Western blot analysis}

The total protein of the MOVAS cells was extracted by RIPA. After the protein denaturation, $20 \mu \mathrm{g}$ of total protein was loaded and separated by using SDS-PAGE at $120 \mathrm{~V}$ for 120 min. Subsequently, the protein was transferred to a Polyvinyl Difluoride (PVDF) transfer membrane (IPVH00010, Millipore) at $4^{\circ} \mathrm{C}$, which then blocked with $5 \%$ non-fat milk at room temperature. Following this, the membranes were incubated with indicated primary antibodies including cyclinD1, CDK4, CDK6, cyclinE2, CDK2, p21 and GAPDH overnight at $4^{\circ} \mathrm{C}$ with gentle shaking. The membranes were washed with TBST, and incubated with secondary antibodies respectively at room temperature with sharking. The protein signals were detected by using ChemiDocTM XRS ${ }^{+}$system (Bio-Rad) $(\mathrm{n}=3)$. And $G A P D H$ was used as a loading control.

\section{Statistical analysis}

Data are expressed as the mean \pm standard error of the mean. Statistical analyses were performed using SPSS version 19.0 software. Comparisons between each group were performed using one-way analysis of variance. $\mathrm{P}<0.05$ was considered to indicate a statistically significant difference. 


\section{Results}

The proliferation of MOVAS cells was inhibited by OMT to identify the role of OMT on VSMCs, we firstly treated the MOVAS cells with different concentrations of OMT to assess the best dosage. Our results of CCK8 showed that the cell proliferation was significantly inhibited by OMT at concentrations of $5 \mu \mathrm{M}, 10 \mu \mathrm{M}, 20 \mu \mathrm{M}, 40 \mu \mathrm{M}$, and no obvious difference was observed between $20 \mu \mathrm{M}$ and $40 \mu \mathrm{M}$ (Figure 1A). Therefore, to lower the potential toxicity of OMT on MOVAS cells, $20 \mu \mathrm{M}$ of OMT was selected for further experiments in this study. Next, MOVAS cells were treated with $20 \mu \mathrm{M}$ of OMT for different times to investigate whether the proliferation inhibition effects of OMT on MOVAS cells is time-dependent manner. Our result demonstrated that the proliferation of MOVAS cells was inhibited by OMT as early as $12 \mathrm{~h}$, and the best inhibition effect was observed at $48 \mathrm{~h}$ (Figure 1B). Thus, in subsequent experiments, we treated the MOVAS cells with $20 \mu \mathrm{M}$ of OMT for 48 hours to study its role and mechanism on VSMCs. As shown in Figure 2A, 20 $\mathrm{ng} / \mathrm{ml}$ of PDGF-BB dramatically promoted the proliferation of MOVAS cells, but the cell number of MOVAS was significantly reduced by $20 \mu \mathrm{M}$ of OMT treatment for $48 \mathrm{~h}$. The CCK8 assay also demonstrated that OMT suppressed PDGF-BB induced MOVAS cells proliferation (Figure 2B). Furthermore, the mRNA levels of proliferation markers PCNA and Ki67 were evaluated by RT-PCR. Compared with DMSO treated group, the expression levels of PCNA and Ki67 were significantly increased in PDGF-BB induced MOVAS cells, but this increment was dampened by OMT treatment (Figure 2C).
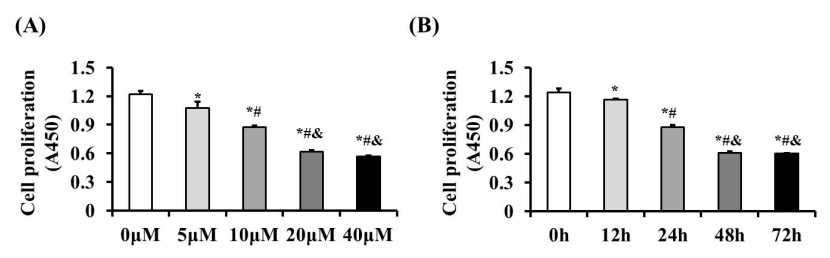

Figure 1. Screening best OMT dosage and treatment time point. (A) The MOVAS cell viability was evaluated by CCK8 assays after treated with different OMT concentrations $(0,5,10,20,40 \mu M)$ for $48 \mathrm{~h}(\mathrm{n}=3),{ }^{*} \mathrm{P}<0.05$ vs. $0 \mu \mathrm{M},{ }^{\#} P<0.05$ vs. $5 \mu \mathrm{M}$, ${ }^{\&} P<0.05$ vs. $10 \mu \mathrm{M}$; (B) The MOVAS cell viability was evaluated by CCK8 assays after treated with $20 \mu M$ of OMT for different times $(0,12,24,48$, and 72 h) $(n=3),{ }^{*} P<0.05$ vs. $0 h,{ }^{\#} P<0.05$ vs. $12 h,{ }^{\&} P<0.05$ vs. $24 h$.

\section{OMT treatment induces MOVAS cell cycle arrest in $G_{0} / G_{1}$ phase}

Cell proliferation is tightly related to cell cycle progression, to elucidate the mechanism of MOVAS proliferation regulated by OMT, we evaluated cell cycle progression of MOVAS. Synchronized with serum starvation, the MOVAS cells were caused cell cycle arrest at $\mathrm{G}_{0} / \mathrm{G}_{1}$, and subsequently labeled with PI and analysed by flow cytometry. It was found that Sphase population was significantly increased and $G_{0} / G_{1}$ phase was significantly decreased in PDGF-BB group, while $G_{0} / G_{1}$ phase of MOVAS in PDGF-BB treated with OMT group was remarkably increased and S-phase population was significantly decreased. These results indicated that OMT treatment induces MOVAS cell cycle arrest in $\mathrm{G}_{0} / \mathrm{G}_{1}$ phase (Table 1 ).

\section{OMT treatment shows no effect on MOVAS apoptosis}

Reduction of cell number may result from inhibition of cell growth or induction of cell apoptosis [26]. To investigate whether OMT induced apoptosis in MOVAS stimulated with PDGF-BB, flow cytometry analysis was applied here. It was showed that there was no significant difference among groups, which indicated that the inhibitory effect of OMT on PDGFBB-stimulated MOVAS might not be associated with cell apoptosis (Figure 3).

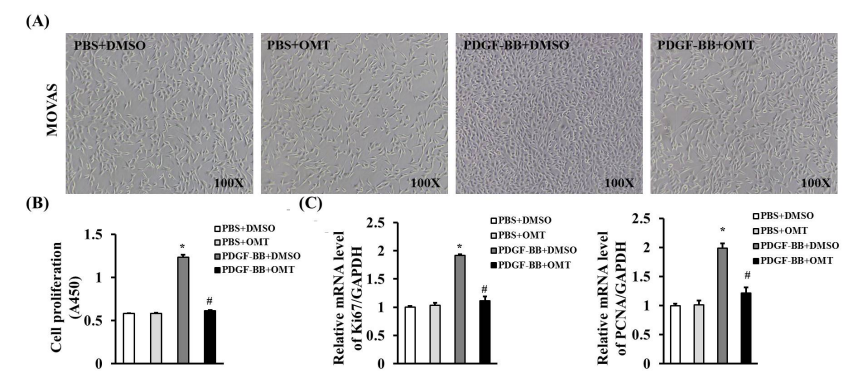

Figure 2. The proliferation of MOVAS cells was inhibited by OMT treatment. (A) Representative images of MOVAS cells under light microscopy.; (B) The proliferation of MOVAS cells was evaluated by CCK8 kit $(n=4)$. (C) The mRNA levels of Ki67 and PCNA were detected by RT-PCR in MOVAS cells with the indicated treatment $(n=4) .{ }^{*} P<0.05$ vs. $P B S+D M S O,{ }^{\#} P<0.05$ vs. $P D G F-B B+D M S O$.

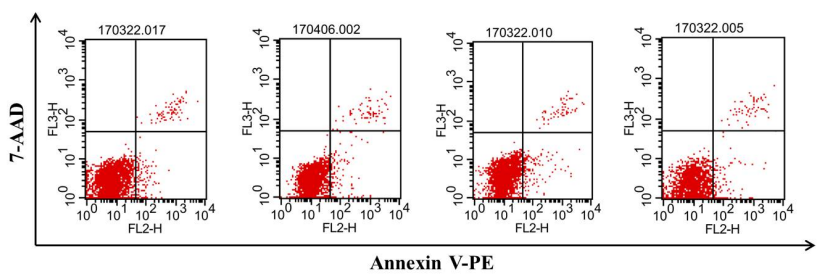

Figure 3. OMT treatment shows no effect on MOVAS apoptosis. The Representative images of MOVAS cells apoptosis evaluated by PE annexin $V$ apoptosis detection kit and detected by flow cytometry $(n=3)$.

\section{OMT inhibits MOVAS enter to $S$ phase via downregulating cyclinD1-CDK4/6, cyclinE2-CDK2 and upregulating p21}

$\mathrm{G}_{0} / \mathrm{G}_{1}$-phase to S-phase transition is primarily regulated by cyclinD1-CDK4/6, cyclinE2-CDK2 [28,29]. Additionally, p21 could downregulates cyclinE2 and CDK2, which contributed into cell cycle arrest [30]. To investigate the levels of these proteins in OMT-treated MOVAS induced by PDGF-BB, the protein levels of them were checked by Western blot. It was shown that the expression levels of cyclinD1, CDK4, CDK6, cyclinE2, and CDK2 were decreased in MOVAS treated with OMT and PDGF-BB, while the level of p21 was increased when comparing to PDGF-BB group, which suggested that 
OMT inhibited the $\mathrm{G}_{1} / \mathrm{S}$-phase transition probably via downregulating cyclinD1-CDK4/6 and cyclinE2-CDK2, and upregulating p21 (Figure 4).

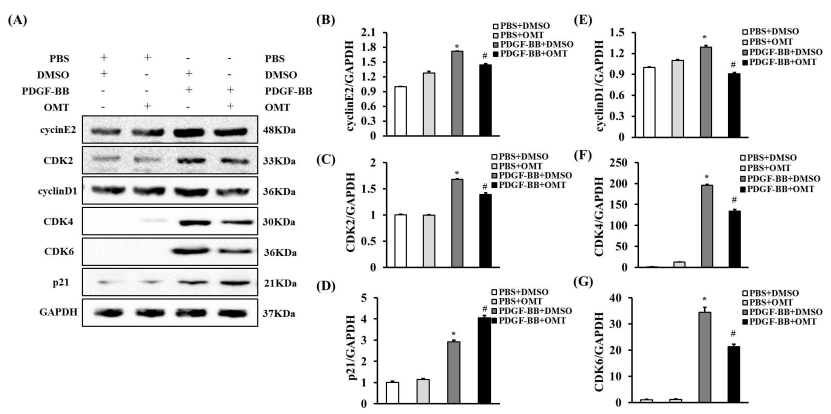

Figure 4. OMT inhibits MOVAS enter to $S$ phase via downregulating cyclinD1-CDK4/6, cyclinE2-CDK2 and upregulating p21. (A) Representative Western blots of the protein levels of cyclinD1, CDK4, CDK6, cyclinE2, CDK2 and 21 in MOVAS cells with the indicated treatment. GAPDH serve as loading control $(n=3) ; \quad(B-G)$ Quantitative result of the levels of cyclinE2, CDK2, p21, cyclinD1, CDK4 and CDK6, respectively. ${ }^{*} P<0.05$ vs. $P B S+D M S O,{ }^{\#} P<0.05$ vs. PDGF-BB+DMSO.

Table 1. OMT treatment induces MOVAS cell cycle arrest in the $G_{0} / G_{1}$-phase (\%).

\begin{tabular}{lllll}
\hline Phase & PBS+DMSO & PBS+OMT & PDGF-BB+DMSO & PDGF-BB+OMT \\
\hline $\mathrm{G}_{0} / \mathrm{G}_{1}$ & $80.08 \pm 0.45$ & $80.70 \pm 0.52$ & $67.03 \pm 0.27^{*}$ & $78.19 \pm 0.71^{\#}$ \\
\hline $\mathrm{S}$ & $19.06 \pm 0.37$ & $18.50 \pm 0.48$ & $32.16 \pm 0.24^{*}$ & $21.05 \pm 0.75^{\#}$ \\
\hline $\mathrm{G}_{2} / \mathrm{M}$ & $0.86 \pm 0.09$ & $0.80 \pm 0.04$ & $0.81 \pm 0.03$ & $0.76 \pm 0.04$ \\
\hline
\end{tabular}

The population of cell cycle phases in each group ( $n=3$ samples per group). ${ }^{*} \mathrm{P}<0.05$ vs. PBS+DMSO, ${ }^{\#} \mathrm{P}<0.05$ vs. PDGF-BB+DMSO.

\section{Discussion}

In the present study, we tried to detect the effect and the probable mechanism of OMT on VSMCs in vitro. We found that: (1) OMT inhibited the proliferation of MOVAS induced by PDGF-BB; (2) OMT induced cell cycle arrest of MOVAS in $\mathrm{G}_{0} / \mathrm{G}_{1}$ phase; (3) OMT showed no effect on MOVAS apoptosis; (4) OMT inhibited the $\mathrm{G}_{1} / \mathrm{S}$-phase transition of MOVAS probably via downregulating cyclinD1-CDK4/6 and cyclinE2-CDK2, and upregulating p21 after PDGF-BB stimulation. Thus, this study firstly found that the antiproliferative effect of OMT on MOVAS through the inhibition of DNA synthesis via $\mathrm{G}_{0} / \mathrm{G}_{1}$ arrest, which indicated that OMT might be used as a novel therapeutic agent to mediate excessive proliferation of VAMCs in the future.

As we known, excessive proliferation of VSMCs is the important pathophysiological process of neointima formation [31-33]. After vascular injury, a variety of growth factors, cytokines and chemokines are synthesized and secreted, which can stimulate VSMCs phenotype switching, accelerate VSMCs migration to inner membranes, induce the excessive proliferation of VSMCs and further lead to vascular restenosis [34-36]. PDGF, mainly expressed in VSMCs and endothelial cells, promote mitosis and play an important role in the proliferation of VSMCs [6-8,37]. The binding of PDGF-BB, a subtype of PDGF released from injured vessels, to its receptor can trigger the activation of downstream and then contribute significantly to VSMCs proliferation [9-12]. In this paper, PDGF-BB could effectively induce MOVAS proliferation, which was consistent with the previous studies reported.

Additionally, it was also found that OMT can significantly suppress MOVAS proliferation induced by PDGF-BB, retard MOVAS in $G_{0} / G_{1}$ phase cannot smoothly into the $S$ phase. The cell cycle is a highly controlled process involved regulatory factors cyclins and CDKs $[36,38]$. In the early of $\mathrm{G}_{0} / \mathrm{G}_{1}$ phase, cyclinD1-CDK4/6 complex can promote $\mathrm{G}_{1}$ transit to $\mathrm{S}$ phase; late in the $\mathrm{G}_{0} / \mathrm{G}_{1}$ phase, cyclinE2 combined with CDK2 also can further promote $G_{1}$ phase enter into $S$ phase [38]. In the present study, it was found that OMT not only inhibited cyclinD1-CDK4/6 expression, but also decreased cyclinE2CDK2 expression. In addition, as we all know, p21 could down-regulate cyclinE2 and CDK2, which is involved in cell cycle arrest [30]. Thus, we detected the expression level of $\mathrm{p} 21$, it was shown that the expression level of $\mathrm{p} 21$ was increased in MOVAS treated with OMT and PDGF-BB when comparing to PDGF-BB group. Collectively, it can be speculated that OMT inhibited MOVAS proliferation induced by PDGF-BB probably through inhibiting the expression of cyclinD1-CDK4/6 and cyclinE2-CDK2 complexes and promoting the expression of $\mathrm{p} 21$, thereby blocked MOVAS in $\mathrm{G}_{0} / \mathrm{G}_{1}$ phase and can't enter into the $\mathrm{S}$ phase.

In conclusion, our results illustrated that OMT inhibited PDGF-BB-induced proliferation of MOVAS via $\mathrm{G}_{0} / \mathrm{G}_{1}$ arrest, resulting from downregulated cyclinD1-CDK4/6 and cyclinE2CDK2, and upregulated p21. Therefore, our study indicated that with its anti-proliferation effect, OMT might be valuable as a therapeutic agent for managing atherosclerosis and vascular restenosis in the future. However, detailed regulatory mechanisms of cyclins-CDKs and p21 involved in the process of OMT affects VAMCs proliferation should be deeply discussed. Additionally, how OMT regulates VAMCs proliferation, atherosclerosis and vascular restenosis remains to be further demonstrated in vivo with animal models.

\section{Acknowledgement}

The authors would like to thank all members of Department of Pediatric cardiovascular medicine, Woman and Child Hospital of Hubei Province (Wuhan, China) for their expert technical assistance and advice.

\section{Conflict of Interest Statement}

The authors declare that they have no conflict of interest.

\section{References}

1. Matsumoto T, Nagayama K. Tensile properties of vascular smooth muscle cells: bridging vascular and cellular biomechanics. J Biomech 2012; 45: 745-755. 
2. Bowen-Pope DF, Ross R, Seifert RA. Locally acting growth factors for vascular smooth muscle cells: endogenous synthesis and release from platelets. Circulation 1985; 72: 735-740.

3. Cao C, Ji X, Luo X, Zhong L. Gingipains from Porphyromonas gingivalis promote the transformation and proliferation of vascular smooth muscle cell phenotypes. Int J Clin Exp Med 2015; 8: 18327-18334.

4. Li J, Zhang M, Ma J. Myricitrin inhibits PDGF-BBstimulated vascular smooth muscle cell proliferation and migration through suppressing PDGFRbeta/Akt/Erk signaling. Int J Clin Exp Med 2015; 8: 21715-21723.

5. Yang G, Zhou X, Chen T, Deng Y, Yu D, Pan S, Song Y. Hydroxy safflor yellow A inhibits lipopolysaccharideinduced proliferation and migration of vascular smooth muscle cells via toll-like receptor-4 pathway. Int J Clin Exp Med 2015; 8: 5295-5302.

6. de Oliveira MG, Doro FG, Tfouni E, Krieger MH. Phenotypic switching prevention and proliferation/ migration inhibition of vascular smooth muscle cells by the ruthenium nitrosyl complex trans-( $\mathrm{Ru}(\mathrm{NO}) \mathrm{Cl}($ cyclam $)$ (PF6 ) 2. J Pharm Pharmacol 2017; 69: 1155-1165.

7. Tan J, Yang L, Liu C, Yan Z. MicroRNA-26a targets MAPK6 to inhibit smooth muscle cell proliferation and vein graft neointimal hyperplasia. Sci Rep 2017; 7: 46602.

8. Pan S, Lin H, Luo H, Gao F, Meng L, Zhou C, Jiang C, Guo Y, Ji Z, Chi J, Guo H. Folic acid inhibits dedifferentiation of PDGF-BB-induced vascular smooth muscle cells by suppressing mTOR/P70S6K signaling. Am J Transl Res 2017; 9: 1307-1316.

9. Lee KP, Lee K, Park WH, Kim H, Hong H. Piperine inhibits platelet-derived growth factor-BB-induced proliferation and migration in vascular smooth muscle cells. J Med Food 2015; 18: 208-215.

10. Cui Y, Sun YW, Lin HS, Su WM, Fang Y, Zhao Y, Wei XQ, Qin YH, Kohama K, Gao Y. Platelet-derived growth factor$\mathrm{BB}$ induces matrix metalloproteinase-2 expression and rat vascular smooth muscle cell migration via ROCK and ERK/p38 MAPK pathways. Mol Cell Biochem 2014; 393: 255-263.

11. Kingsley K, Plopper GE. Platelet-derived growth factor modulates rat vascular smooth muscle cell responses on laminin-5 via mitogen-activated protein kinase-sensitive pathways. Cell Commun Signal 2005; 3: 2.

12. Kamimura M, Bea F, Akizawa T, Katus HA, Kreuzer J, Viedt C. Platelet-derived growth factor induces tissue factor expression in vascular smooth muscle cells via activation of Egr-1. Hypertension 2004; 44: 944-951.

13. Park HS, Quan KT, Han JH, Jung SH, Lee DH, Jo E, Lim TW, Heo KS, Na MK, Myung CS. Rubiarbonone C inhibits platelet-derived growth factor-induced proliferation and migration of vascular smooth muscle cells through the FAK, MAP kinase, and STAT3 Tyr705 signaling pathways. Br J Pharmacol 2017.
14. Lu ML, Xiang XH, Xia SH. Potential signaling pathways involved in the clinical application of oxymatrine. Phytother Res 2016; 30: 1104-1112.

15. Ho JW, Ngan Hon PL, Chim WO. Effects of oxymatrine from $\mathrm{Ku}$ Shen on cancer cells. Anticancer Agents Med Chem 2009; 9: 823-826.

16. Jiang Y, Zhu Y, Mu Q, Luo H, Zhi Y, Shen X. Oxymatrine provides protection against Coxsackievirus B3-induced myocarditis in BALB/c mice. Antiviral Res 2017; 141: 133-139.

17. Liu Y, Bi T, Wang Z, Wu G, Qian L, Gao Q, Shen G. Oxymatrine synergistically enhances antitumor activity of oxaliplatin in colon carcinoma through PI3K/AKT/mTOR pathway. Apoptosis 2016; 21: 1398-1407.

18. Ma A, Yang Y, Wang Q, Wang Y, Wen J, Zhang Y. Antiinflammatory effects of oxymatrine on rheumatoid arthritis in rats via regulating the imbalance between Treg and Th17 cells. Mol Med Rep 2017; 15: 3615-3622.

19. Chen Q, Zhou H, Yang Y, Chi M, Xie N. Investigating the potential of oxymatrine as a psoriasis therapy. Chem Biol Interact 2017; 271: 59-66.

20. Runtao G, Guo D, Jiangbo Y, Xu W, Shusen Y. Oxymatrine, the main alkaloid component of Sophora roots, protects heart against arrhythmias in rats. Planta Med 2011; 77: 226-230.

21. Cao YG, Jing S, Li L, Gao JQ, Shen ZY, Liu Y, Xing Y, Wu ML, Wang Y, Xu CQ, Sun HL. Antiarrhythmic effects and ionic mechanisms of oxymatrine from Sophora flavescens. Phytother Res 2010; 24: 1844-1849.

22. Hong-Li S, Lei L, Lei S, Dan Z, De-Li D, Guo-Fen Q, Yan L, Wen-Feng C, Bao-Feng Y. Cardioprotective effects and underlying mechanisms of oxymatrine against Ischemic myocardial injuries of rats. Phytother Res 2008; 22: 985-989.

23. Zhang W, Zhang J, Liu YK, Liu J, Wang X, Xu Q, Wang Y, $\mathrm{Xu} \mathrm{X}$, Dai G. Cardioprotective effects of oxymatrine on isoproterenol-induced heart failure via regulation of DDAH/ADMA metabolism pathway in rats. Eur $\mathbf{J}$ Pharmacol 2014; 745: 29-35.

24. Huang XY, Chen CX. Effect of oxymatrine, the active component from Radix Sophorae flavescentis (Kushen), on ventricular remodeling in spontaneously hypertensive rats. Phytomedicine 2013; 20: 202-212.

25. Li G, Liu S, Yang Y, Xie J, Liu J, Kong F, Tu G, Wu R, Li G, Liang S. Effects of oxymatrine on sympathoexcitatory reflex induced by myocardial ischemic signaling mediated by $\mathrm{P} 2 \mathrm{X}(3)$ receptors in rat SCG and DRG. Brain Res Bull 2011; 84: 419-424.

26. Mao H, Tao T, Song D, Liu M, Wang X, Liu X, Shi D. Zedoarondiol inhibits platelet-derived growth factorinduced vascular smooth muscle cells proliferation via regulating AMP-activated protein kinase signaling pathway. Cell Physiol Biochem 2016; 40: 1506-1520.

27. Lee SJ, Won SY, Park SL, Song JH, Noh DH, Kim HM, Yin CS, Kim WJ, Moon SK. Rosa hybrida extract suppresses vascular smooth muscle cell responses by the 
targeting of signaling pathways, cell cycle regulation and matrix metalloproteinase-9 expression. Int $\mathrm{J}$ Mol Med 2016; 37: 1119-1126.

28. Zetterberg A, Larsson O, Wiman KG. What is the restriction point? Curr Opin Cell Biol 1995; 7: 835-842.

29. Yi X, Tao Y, Lin X, Dai Y, Yang T, Yue X, Jiang X, Li X, Jiang DS, Andrade KC, Chang J. Histone methyltransferase Setd2 is critical for the proliferation and differentiation of myoblasts. Biochim Biophys Acta 2017; 1864: 697-707.

30. Harper JW, Elledge SJ, Keyomarsi K, Dynlacht B, Tsai LH, Zhang P, Dobrowolski S, Bai C, Connell-Crowley L, Swindell E. Inhibition of cyclin-dependent kinases by $\mathrm{p} 21$. Mol Biol Cell 1995; 6: 387-400.

31. Huang SC, Wang M, Wu WB, Wang R, Cui J, Li W, Li ZL, Li W, Wang SM. Mir-22-3p inhibits arterial smooth muscle cell proliferation and migration and neointimal hyperplasia by targeting HMGB1 in arteriosclerosis obliterans. Cell Physiol Biochem 2017; 42: 2492-2506.

32. Rani K, Aung NY. Docosahexaenoic acid inhibits vascular smooth muscle cell proliferation induced by glucose variability. Open Biochem J 2017; 11: 56-65.

33. Kim JH, Bae KH, Byun JK, Lee S, Kim JG, Lee IK, Jung GS, Lee YM, Park KG. Lactate dehydrogenase-A is indispensable for vascular smooth muscle cell proliferation and migration. Biochem Biophys Res Commun 2017; 492: 41-47.

34. Wu B, Mottola G, Schaller M, Upchurch GR, Conte MS. Resolution of vascular injury: Specialized lipid mediators and their evolving therapeutic implications. Mol Aspects Med 2017.

35. Yang J, Fan Z, Yang J, Ding J, Yang C, Chen L. MicroRNA-24 attenuates neointimal hyperplasia in the diabetic rat carotid artery injury model by inhibiting Wnt4 signaling pathway. Int J Mol Sci 2016; 17.

36. Jun MY, Karki R, Paudel KR, Sharma BR, Adhikari D, Kim DW. Alkaloid rich fraction from Nelumbo nucifera targets VSMC proliferation and migration to suppress restenosis in balloon-injured rat carotid artery. Atherosclerosis 2016; 248: 179-189.

37. Peng C, Zhang S, Liu H, Jiao Y, Su G, Zhu Y. A newly synthesized Ligustrazine stilbene derivative inhibits PDGFBB induced vascular smooth muscle cell phenotypic switch and proliferation via delaying cell cycle progression. Eur J Pharmacol 2017.

38. Fisher RP. Getting to S: CDK functions and targets on the path to cell-cycle commitment. F1000Res 2016; 5: 2374.

\section{*Correspondence to}

Xia Gao

Department of Pathology

Integrated Chinese and Western Medicine Hospital of Hubei Provincal

PR China 